\title{
Long-term outcomes of resective epilepsy surgery after invasive presurgical evaluation in children with tuberous sclerosis complex and bilateral multiple lesions
}

\author{
Ravindra Arya, MD, DM, ${ }^{1}$ Jeffrey R. Tenney, MD, PhD, ${ }^{1}$ Paul S. Horn, PhD, ${ }^{1}$ Hansel M. Greiner, MD, ${ }^{1}$ \\ Katherine D. Holland, MD, PhD, ${ }^{1}$ James L. Leach, MD, ${ }^{2}$ Michael J. Gelfand, MD, ${ }^{3}$ \\ Leonid Rozhkov, MS, ${ }^{1}$ Hisako Fujiwara, REEGT,1 Douglas F. Rose, MD,, David N. Franz, MD,1 \\ and Francesco T. Mangano, $\mathrm{DO}^{4}$
}

\begin{abstract}
Divisions of ${ }^{1}$ Neurology, ${ }^{2}$ Pediatric Neuroradiology, ${ }^{4}$ Pediatric Neurosurgery, and ${ }^{3}$ Nuclear Medicine Section, Department of Radiology, Cincinnati Children's Hospital Medical Center, Cincinnati, Ohio
\end{abstract}

\begin{abstract}
OBJECT Tuberous sclerosis complex (TSC) with medically refractory epilepsy is characterized by multifocal brain abnormalities, traditionally indicating poor surgical candidacy. This single-center, retrospective study appraised seizurerelated, neuropsychological, and other outcomes of resective surgery in TSC patients with medically refractory epilepsy, and analyzed predictors for these outcomes.

METHODS Patients with multilesional TSC who underwent epilepsy surgery between 2007 and 2012 were identified from an electronic database. All patients underwent multimodality noninvasive and subsequent invasive evaluation. Seizure outcomes were classified using the International League Against Epilepsy (ILAE) scale. The primary outcome measure was complete seizure remission (ILAE Class 1 ). Secondary outcome measures included $50 \%$ responder rate, change in full-scale IQ, electroencephalography improvement, and reduction in antiepileptic drug (AED) burden.

RESULTS A total of 37 patients with TSC underwent resective surgery during the study period. After a mean follow-up of $5.68 \pm 3.67$ years, $56.8 \%$ achieved complete seizure freedom (ILAE Class 1 ) and $86.5 \%$ had ILAE Class 4 outcomes or better. The full-scale IQ on follow-up was significantly higher in patients with ILAE Class 1 outcome $(66.70 \pm 12.36)$ compared with those with ILAE Class 2 or worse outcomes $(56.00 \pm 1.41, p=0.025)$. In $62.5 \%$ of the patients with ILAE Class 2 or worse outcomes, the number of AEDs were found to be significantly reduced $(p=0.004)$.

CONCLUSIONS This study substantiates the evidence for efficacy of resective epilepsy surgery in patients with bilateral multilesional TSC. More than half of the patients were completely seizure free. Additionally, a high proportion achieved clinically meaningful reduction in seizure burden and the number of AEDs.
\end{abstract}

http://thejns.org/doi/abs/10.3171/2014.10.PEDS14107

KEY WORDS epilepsy surgery; medically refractory epilepsy; tuberous sclerosis complex

$\mathrm{T}$ Uuberous sclerosis complex (TSC) is a multisystem genetic disease with a wide spectrum of neurological disability caused by epilepsy, cognitive impairment, and autism. Epilepsy occurs in more than $80 \%$ of patients with TSC, with nearly two-thirds of cases refractory to medical management. ${ }^{13}$ The pathophysiology of epilepsy and mechanisms of resistance or refractoriness to antiepileptic drugs (AEDs) are not well understood. It is uncertain if tubers, which represent areas of dysgenesis, are themselves epileptogenic or influence neighboring neuronal networks to generate seizures. ${ }^{11}$ It is further uncertain if all tubers are equivalent in terms of seizure genesis or whether certain tuber characteristics reliably predict occurrence, type(s), or burden of seizures in an individual patient. However, the association of medically refractory epilepsy in TSC with increased prevalence of intellectual

ABBREVIATIONS AED = antiepileptic drug; ECD = equivalent current dipole; ECoG = electrocorticography; EEG = electroencephalography; FSIQ = full-scale IQ; ILAE = International League Against Epilepsy; IQR = interquartile range; MEG = magnetoencephalography; SPM = statistical parametric mapping; TSC = tuberous sclerosis complex.

SUBMITTED March 2, 2014. ACCEPTED October 2, 2014.

INCLUDE WHEN CITING Published online October 31, 2014; DOI: 10.3171/2014.10.PEDS14107.

DISCLOSURE The authors report no conflict of interest concerning the materials or methods used in this study or the findings specified in this paper. 
disability and psychobehavioral morbidity has been well recognized. ${ }^{6,13}$ Hence, such patients are usually evaluated for epilepsy surgery in an attempt to reduce seizure burden. Traditionally, resective epilepsy surgery has been driven by conventional criteria of multimodality concordance and has been usually limited to patients having a single, well-localized tuber with a corresponding seizure onset zone. Other patients have either been denied surgery or offered only palliative procedures because a poor outcome was anticipated. ${ }^{3}$ There is growing concern about this intrinsic bias, and current epilepsy surgery in TSC is moving toward more extensive resections driven by concordance of imaging, neurophysiological, and other data. To substantiate the evidence in favor of such a data-driven approach, we present a single-center experience with resective surgery in TSC patients with medically refractory epilepsy who harbored multiple bilateral brain lesions.

\section{Methods \\ Patient Population}

In this single-center retrospective chart review study patients were identified from an institutional epilepsy surgery electronic database. All patients with TSC who underwent resective epilepsy surgery between January 2007 and December 2012 were reviewed for inclusion. The diagnoses of TSC and medical refractoriness were based on conventional criteria., ${ }^{9,12}$ The patients were classified as having bilateral disease if the MRI study demonstrated tubers or areas of cortical signal abnormality consistent with cortical dysplasia in both hemispheres.

\section{Presurgical Evaluation}

A comprehensive noninvasive presurgical evaluation including a detailed history; systematic and neurological examination; and neuropsychological, neurophysiological, and imaging studies was individualized for all patients by the attending epileptologist (Fig. 1). A 5-day video-electroencephalography (EEG) study capturing a sufficient number of habitual electroclinical seizures was performed, and both interictal and ictal electrographic changes were analyzed visually. Structural imaging consisted of an epilepsy protocol MRI study. Functional imaging comprised interictal FDG-PET, ictal and interictal SPECT, and magnetoencephalography (MEG) studies. Areas of relative hypometabolism were determined by statistical parametric mapping (SPM) of PET data, in addition to visual analysis of raw PET data. Interictal SPECT was digitally subtracted from the ictal study and coregistered on MRI (subtraction ictal SPECT coregistered to MRI [SISCOM]) to determine area(s) of relative hyperperfusion during a seizure. MEG data were obtained with concurrent scalp EEG data and different interictal spikes or ictal onset, if captured. Although MEG data were modeled for source localization using various algorithms, for the purposes of this study only the equivalent current dipole (ECD) findings were analyzed.

Information obtained from different modalities in the noninvasive evaluation was synthesized for each patient individually and was discussed in a multidisciplinary conference that included specialists in neurology, neurosur- gery, neuroimaging, nuclear medicine, neuropsychology, nursing, and social work. In patients with bilateral ictal onset on EEG or if the functional imaging (PET, SPECT, and MEG) findings were discordant with EEG ictal onset, the decision regarding invasive evaluation was complex. For example, if the majority of seizures were lateralized to one hemisphere with one or relatively fewer seizures from the other hemisphere or if one of the functional modalities was discordant but others agreed with EEG ictal onset, then the individual decisions were guided by the bulk of the evidence.

\section{Surgical Technique}

For all patients included in this report, a decision was made for invasive monitoring using subdural electrodes. The first craniotomy exposed the lobes of interest using standard techniques. In the majority of patients, the head was placed on a horseshoe-type holder without the need for cranial fixation. Once the dura was opened, an intraoperative photograph was obtained, and electrode grids and strips were laid over the area(s) of interest under electrocorticography (ECoG) monitoring. After agreement about coverage, electrode leads were sutured to the dura and a second intraoperative photograph was obtained. The dura was closed with a temporal based patch if indicated. All patients had a subgaleal drain placed, with leads additionally sutured to the galea and skin on the exit site. The bone was replaced with a plate and 2 screws at the superior portion of the craniotomy. The patient's head was dressed and wrapped with leads and a drain exiting from the vertex. The patient was woken up, extubated, and monitored in the intensive care unit for 24 hours before being transferred to the epilepsy monitoring unit. All patients underwent postoperative CT scanning of the head with $3 \mathrm{D}$ reconstructions of grid placement for verification of location of the subdural electrodes. After a sufficient number of habitual electro-clinical seizures were captured, localization of the central sulcus with somatosensory evoked potentials and functional mapping of motor and/or language cortices with extraoperative cortical stimulation was performed as indicated. The data were again synthesized, and a resection plan was formulated by the epilepsy team (Fig. 1). Additionally, image guidance or intraoperative motor mapping was used particularly if proposed resections were adjacent to eloquent cortices. No patient in this study underwent awake craniotomy.

During the resection, surgical margins were extended to include whole tubers if the ECoG study identified a part of the tuber (or tuberous region) to be active at seizure onset. Postresection ECoG was performed, and resection margins were modified on an individual basis if electrographic seizures, runs of spikes, or frequent bursts of highfrequency oscillations were seen.

\section{Outcome Measures}

Seizure outcome was classified according to the International League Against Epilepsy (ILAE) outcome scale. ${ }^{16}$ ILAE outcome classification is an ordinal scale based on seizure-days/year, with categories defined as complete seizure freedom without auras (Class 1), only auras without 

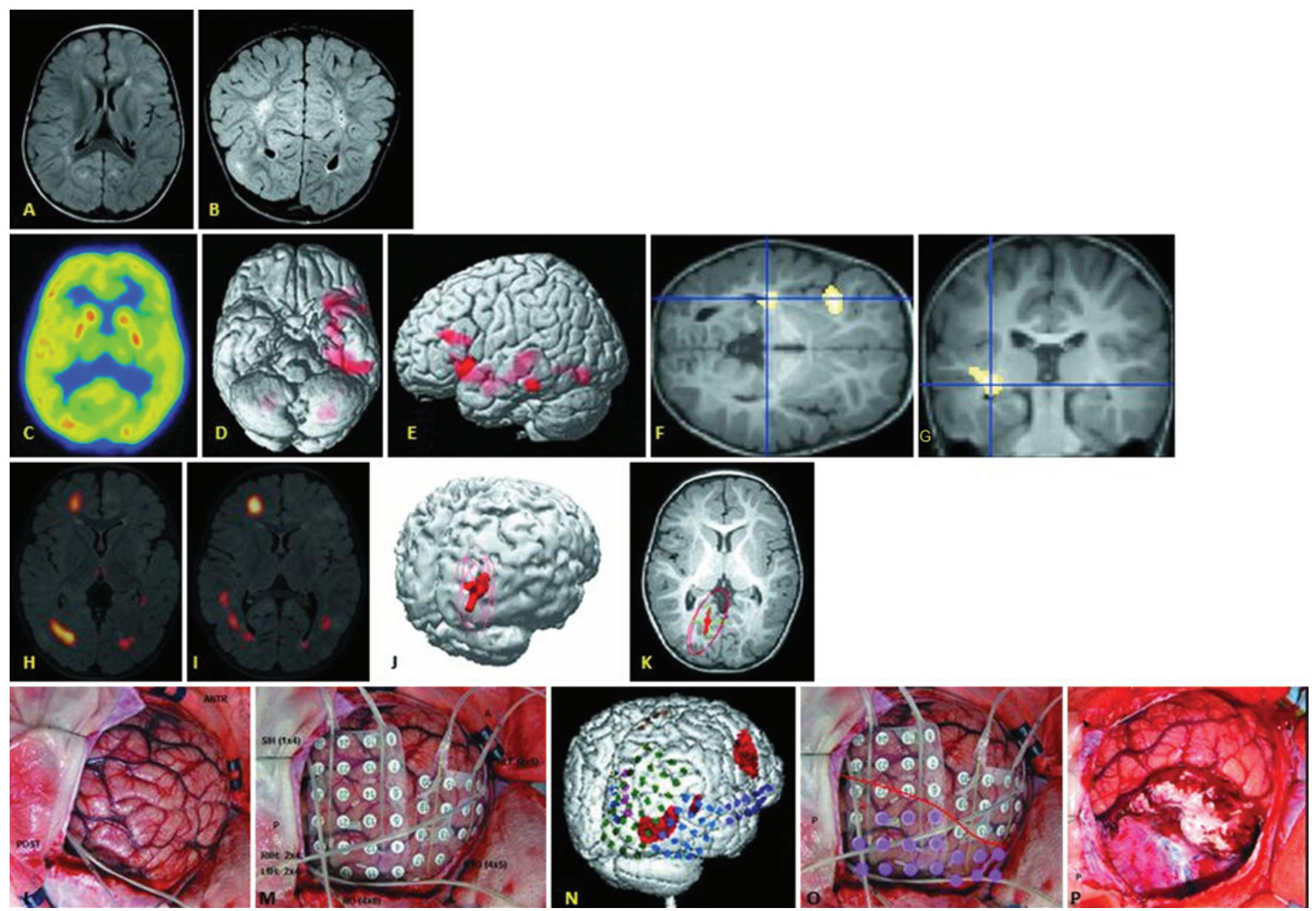

FIG. 1. Illustrative case. This 5-year-old girl began having seizures at 3 months of age, characterized by head drops, eye rolling, and stiffening of the whole body, followed by crying. By 2 years of age, she also developed another seizure type with leftward gaze deviation. At the time of surgical referral, 4 antiseizure medications had failed and she had mild global delay. EEG showed bilateral multifocal epileptiform abnormalities, with bilateral occipital (more frequently right-sided lead-in) seizure onsets. MRI showed bilateral multiple areas of signal changes and a subependymal nodule near the left frontal horn (A and B). FDG-PET showed multiple focal areas of decreased metabolism (C). On SPM, there was a prominent area of hypometabolism involving the left frontal, temporal, and anterior parietal lobes (D-G), with less significant hypometabolic area in the right anterior parietal lobe. SISCOM detected areas of relative hyperperfusion in the right occipital, posterior parietal, and frontal lobes ( $\mathrm{H}$ and $\mathrm{I})$. ECD-MEG found the source in the right occipital lobe ( $\mathrm{J}$ and $\mathrm{K}$ ). In view of EEG, SISCOM, and MEG concordance, a decision was made for a right parietooccipital craniotomy (L), despite nonlateralizing MRI and PET. Subdural grids covering right occipital and posterior parietal lobes, along with interhemispheric strips, were placed for invasive monitoring (M and $\mathbf{N}$ ). Finally, a right occipital lobectomy was done with postresection electrocorticography to define resection margins $(\mathbf{O}$ and $\mathrm{P})$. The patient remains completely seizure free at the 2-year follow-up. Figure is available in color online only.

other seizures (Class 2), 1-3 seizure-days/year (Class 3), 4 seizure-days/year to a decrease of $50 \%$ from pretreatment seizure frequency (Class 4), from $50 \%$ decrease up to $100 \%$ increase (Class 5), and $\geq 100 \%$ increase in seizure frequency (Class 6).

The primary outcome measure was complete seizure freedom (ILAE Class 1). Secondary outcomes included the proportion of patients attaining ILAE outcome Class 4 or better, neuropsychological improvement measured by change in full-scale IQ (FSIQ) at last follow-up compared with baseline, improvement in follow-up EEG findings based on routine visual analysis in patients who did not achieve seizure freedom, and change in the AED burden from presurgery visit to last follow-up. Seizure frequency was ascertained from the patient's electronic medical record, where it is prospectively recorded at each follow-up visit by parental questioning.

\section{Statistical Analysis}

Data were extracted onto an Excel spreadsheet (Microsoft Corp.). Variables were scanned for distribution. Means and standard deviations were calculated for normally distributed variables, whereas medians and interquartile ranges (IQRs) were calculated in case of skewed distribution. Planned comparisons between appropriate groups were carried out for the above outcome measures. Means, medians, and proportions were compared using ttests for independent variables, Wilcoxon sign-rank test, 
and Fisher's exact test, respectively. All comparisons were considered significant at a 2 -sided $p$ value of $\leq 0.05$. SAS statistical software (version 9.3, SAS Institute Inc.) was used for statistical analyses. The study was approved by institutional review board of Cincinnati Children's Hospital Medical Center.

\section{Results \\ Patient Characteristics}

Between 2007 and 2012, 37 patients with TSC (21 females, 56.8\%) underwent resective surgery for medically refractory epilepsy (Table 1$)$. In 31 patients $(83.8 \%)$ seizures started during infancy, with the mean age at seizure onset for the entire cohort being $8.81 \pm 14$ months. At the time of referral for surgery, the baseline seizure frequency varied widely from a seizure every 3-4 months to hundreds of seizures per day (median 5 seizures/day, IQR 8 seizures/day). The mean age at the time of surgery was $6.16 \pm 5.96$ years, with a mean preoperative duration of epilepsy of $5.52 \pm 5.87$ years. The preoperative FSIQ was available for 23 patients, with 13 patients $(56.5 \%)$ having FSIQs of 70 or lower (median 58, IQR 31.5). A median of 6 AEDs (IQR 3) failed per patient by the time of surgical referral. All patients in this series had multiple bilateral tubers on brain MRI.

During noninvasive presurgical evaluation, only 14 of 34 patients $(41.2 \%)$ showed ictal onset from a single or 2 ipsilateral contiguous head regions on EEG monitoring (Table 1). In 18 of 26 patients (69.2\%) and 8 of 22 patients (36.4\%), respectively, SPM-PET and SISCOM/ ictal-SPECT findings were concordant with ictal onset on EEG. Source imaging for interictal epileptiform discharges using ECD algorithm with MEG data were concordant with ictal onset on EEG in 13 of 20 patients (65\%). All 3 functional modalities were concordant with each other in 8 of 20 patients (38.1\%).
All patients included in this report underwent invasive monitoring with subdural electrodes. The number of implanted electrodes varied from 44 to 112 (mean $84 \pm 17$ ). Few patients had interhemispheric strips with bilateral recording surfaces $(n=3)$, or contralateral strips inserted through a bur hole $(\mathrm{n}=1)$, in addition to subdural electrodes placed via a craniotomy.

\section{Seizure Outcomes}

After a mean follow-up of $5.68 \pm 3.67$ years (minimum 2.25 years), 21 patients (56.8\%) were completely seizure free (ILAE Class 1 outcome, Table 1). Thirty-two patients $(86.5 \%)$ had an ILAE outcome of Class 4 or better. No patient had worsening of seizures. No predictors could be identified among demographic and clinical variables for seizure remission in these patients (Table 1). Although there was no significant difference between the two seizure outcome groups by the type of resective surgeries, extratemporal lobectomy was more commonly performed in those with ILAE Class 2 or worse outcomes (Fig. 2).

\section{Neuropsychological Outcomes}

The follow-up FSIQ was observed to be significantly higher in patients with complete seizure remission (ILAE Class 1 outcome, $66.70 \pm 12.36$ ) than in those with ILAE Class 2 or worse outcomes $(56.00 \pm 1.41, p=0.025$, Table 1). Both preoperative and follow-up FSIQ scores were available for 9 patients and showed a median gain of 3 points (IQR 9). This change in IQ was found to have a significant positive correlation with age of the patient (Pearson's coefficient $0.712, \mathrm{p}=0.031$, Table 2).

\section{Other Outcomes}

In 6 patients $(40 \%)$ without seizure remission (ILAE class $\geq 2$ ), video-EEG findings were observed to have improved on follow-up based only on routine visual analysis.

TABLE 1. Univariate comparison of demographic and other clinical variables for patients with (ILAE Class 1) and without (ILAE Class $\geq 2$ ) complete seizure freedom*

\begin{tabular}{|c|c|c|c|c|}
\hline Variable & All Patients & ILAE Class 1 & ILAE Class $\geq 2$ & $\mathrm{p}$ Value \\
\hline Mean age at seizure onset in mos & $8.81 \pm 13.95$ & $11.19 \pm 17.80(n=21)$ & $5.47 \pm 3.62(n=15)$ & 0.166 \\
\hline Mean presurgery daily seizure frequency & $11.98 \pm 19.42$ & $16.56 \pm 24.63(n=21)$ & $5.98 \pm 5.30(n=16)$ & 0.069 \\
\hline Mean presurgery FSIQ & $68.17 \pm 19.43$ & $69.00 \pm 18.41(n=14)$ & $66.89 \pm 22.01(n=9)$ & 0.814 \\
\hline Mean no. of electrodes & $83.96 \pm 17.09$ & $81.27 \pm 19.33(n=15)$ & $87.64 \pm 13.47(n=11)$ & 0.332 \\
\hline Mean age at the time of surgery in yrs & $6.16 \pm 5.96$ & $5.54 \pm 4.52(n=21)$ & $6.97 \pm 7.53(n=16)$ & 0.507 \\
\hline Mean duration from onset of seizures to surgery in yrs & $5.52 \pm 5.87$ & $4.61 \pm 4.20(n=21)$ & $6.80 \pm 7.61(n=15)$ & 0.324 \\
\hline Mean duration of follow-up in yrs & $5.68 \pm 3.67$ & $5.64 \pm 3.69(n=18)$ & $5.73 \pm 3.75(n=14)$ & 0.948 \\
\hline Mean follow-up FSIQ & & $66.70 \pm 12.36(n=10)$ & $56.00 \pm 1.41(n=2)$ & 0.025 \\
\hline \multicolumn{5}{|l|}{ No. of patients } \\
\hline No. of females & $21 / 37(56.8 \%)$ & $14 / 21(66.67 \%)$ & $7 / 16(43.75 \%)$ & 0.1964 \\
\hline $\begin{array}{l}\text { EEG ictal onset from } 1 \text { or } 2 \text { ipsilateral contiguous head } \\
\text { regions }\end{array}$ & $14 / 34(41.2 \%)$ & $9 / 19(47.37 \%)$ & $5 / 15(33.33 \%)$ & 0.2926 \\
\hline SPM/PET concordance w/ ictal EEG & $18 / 26(69.2 \%)$ & $11 / 14(78.57 \%)$ & $7 / 12(58.33 \%)$ & 0.4009 \\
\hline SISCOM concordance w/ ictal EEG & $8 / 22(36.4 \%)$ & $4 / 10(40.00 \%)$ & $4 / 12(33.33 \%)$ & 0.9999 \\
\hline MEG concordance w/ ictal EEG & $13 / 20(65 \%)$ & $7 / 11(63.64 \%)$ & $6 / 9(66.67 \%)$ & 0.9999 \\
\hline All functional modalities concordant & $8 / 20(38.1 \%)$ & $5 / 12(41.67 \%)$ & $3 / 9(33.33 \%)$ & 0.9999 \\
\hline
\end{tabular}

\footnotetext{
${ }^{*}$ Mean values are presented as the mean $\pm \mathrm{SD}$. Values in boldface are statistically significant.
} 




FIG. 2. Comparison of 2 outcome groups by the type of resective epilepsy surgery. $A H=$ amygdalohippocampectomy; $A T L=$ anterior temporal lobectomy. Figure is available in color online only.

The improvement was inferred from absence of or reduction in the frequency and extent of electrographic seizures and/or interictal epileptiform discharges. No statistically significant clinical predictors for improvement in EEG could be found (Table 3). The groups with lateralizing and nonlateralizing ictal onsets on video-EEG were comparable (Table 4).

The number of AEDs was found to be significantly reduced after surgery in patients who did not achieve seizure freedom $(p=0.004)$. The number of AEDs was reduced by $1-3$ in 10 patients $(62.5 \%)$ during follow-up, whereas it remained the same in 5 patients and increased in 1 patient (Fig. 3).

\section{Postoperative Complications}

Five patients (13.5\%) had complications during the postoperative period, all of which caused transient problems that resolved without any permanent adverse neurological consequences. The complications included pseudomeningocele $(n=2)$ and wound infection with CSF leak, transient right hemiparesis for 24 hours on the 2 nd postoperative day, and deep venous thrombosis ( $n=1$ each).

\section{Discussion}

This large, single-center experience with resective epilepsy surgery in patients with multilesional TSC substantiates the evidence in support of the efficacy and safety of this approach. More than half (56.8\%) of the patients were completely seizure free and $86.5 \%$ had a reduction of $50 \%$ or more in seizure frequency in this cohort at a mean follow-up of 5.68 years. This is in agreement with a recent meta-analysis that reported $59 \%$ of patients to be seizure free. ${ }^{17}$ This meta-analysis included 13 studies $(\mathrm{n}=$ 229), 12 of which included 25 patients or fewer with only one study having 70 patients collected from 6 different centers..$^{10,17}$ The majority of smaller studies in the metaanalysis reported higher rates for seizure freedom, while the larger study $(n=70)$ reported a seizure-free rate of $53 \%{ }^{10}$ Follow-up details were not provided for individual studies. ${ }^{17}$ Another individual-patient-data meta-analysis of 20 studies with 181 participants found that $56 \%$ of patients had an Engel Class I outcome. ${ }^{5}$ This meta-analysis had somewhat broader inclusion criteria with individual studies comprising 3-25 patients.

TABLE 2. Correlation of different clinical variables with change in FSIQ from baseline presurgery value to that on last available follow-up

\begin{tabular}{lcc}
\hline \multicolumn{1}{c}{ Variable } & Pearson's Correlation Coefficient & $p$ Value $^{*}$ \\
\hline Age in yrs $(n=9)$ & 0.712 & 0.031 \\
\hline Age of seizure onset in mos $(n=9)$ & 0.170 & 0.661 \\
\hline Presurgery daily seizure frequency $(n=9)$ & 0.164 & 0.674 \\
\hline Presurgery FSIQ $(n=9)$ & -0.605 & 0.084 \\
\hline No. of electrodes $(n=7)$ & -0.451 & 0.310 \\
\hline Age at the time of surgery in yrs $(n=9)$ & 0.401 & 0.285 \\
\hline Duration from onset of seizures to surgery in yrs $(n=9)$ & 0.425 & 0.254 \\
\hline Duration of follow-up in yrs $(n=9)$ & 0.535 & 0.138 \\
\hline Follow-up FSIQ $(n=9)$ & 0.179 & 0.645 \\
\hline
\end{tabular}

* The value in boldface is statistically significant. 
TABLE 3. Univariate analysis for improvement in follow-up EEG of patients who did not attain seizure freedom (ILAE Class $\geq 2)^{*}$

\begin{tabular}{lccc}
\hline \multicolumn{1}{c}{ Variable } & $\begin{array}{c}\text { Follow-Up EEG } \\
\text { Improved }\end{array}$ & $\begin{array}{c}\text { Follow-Up EEG Did Not } \\
\text { Improve/Worsened }\end{array}$ & $p$ Value \\
\hline Mean age in yrs & $11.44 \pm 6.63(n=6)$ & $14.21 \pm 8.45(n=9)$ & 0.491 \\
\hline Mean age at seizure onset in mos & $7.33 \pm 4.63(n=6)$ & $4.63 \pm 2.07(n=8)$ & 0.227 \\
\hline Mean presurgery daily seizure frequency & $6.55 \pm 7.18(n=6)$ & $5.81 \pm 4.45(n=9)$ & 0.828 \\
\hline Mean presurgery FSIQ & $69.33 \pm 27.10(n=3)$ & $65.67 \pm 21.81(n=6)$ & 0.850 \\
\hline Mean no. of electrodes & $95.20 \pm 10.06(n=5)$ & $80.80 \pm 14.81(n=5)$ & 0.115 \\
\hline Mean age at the time of surgery in yrs & $5.49 \pm 6.46(n=6)$ & $8.10 \pm 8.78(n=9)$ & 0.518 \\
\hline Mean duration from onset of seizures to surgery in yrs & $4.88 \pm 6.26(n=6)$ & $8.39 \pm 9.03(n=8)$ & 0.407 \\
\hline Mean duration of follow-up in yrs & $5.60 \pm 4.32(n=6)$ & $5.46 \pm 3.69(n=7)$ & 0.954 \\
\hline No. of patients & & & $3 / 9(33.33 \%)$ \\
\hline No. of females & $4 / 6(66.67 \%)$ & $3 / 8(37.50 \%)$ & 0.3147 \\
\hline EEG ictal onset from a single or 2 ipsilateral contiguous & $2 / 6(33.33 \%)$ & & 0.9999 \\
$\quad$ head regions & & $3 / 7(42.86 \%)$ & 0.2929 \\
\hline SPM/PET concordance $w /$ ictal EEG & $4 / 5(80.00 \%)$ & $2 / 7(28.57 \%)$ & 0.9999 \\
\hline SISCOM concordance $w /$ ictal EEG & $2 / 5(40.00 \%)$ & $4 / 5(80.00 \%)$ & 0.5238 \\
\hline MEG concordance w/ ictal EEG & $2 / 4(50.00 \%)$ & $2 / 5(40.00 \%)$ & 0.9999 \\
\hline All functional modalities concordant & $1 / 4(25.00 \%)$ & & \\
\hline
\end{tabular}

* Mean values are presented as the mean \pm SD.

In TSC-associated medically refractory epilepsy, seizure freedom may not be the only representative outcome. Hence, we explored the group with ILAE Class 2 or worse outcomes for further indices of improvement. Epilepsy surgery was found to significantly reduce the AED burden in $62.5 \%$ of these patients (Fig. 3). The decision to modify the AED regimen for individual patients was at the discretion of the treating neurologist, usually based on reduced seizure frequency for a continuous period of 6-12 months. Since poly-pharmacy in patients with epilepsy is a major risk factor for adverse effects and contributes to morbidity, ${ }_{1}$ a decrease in the number of AEDs even in patients who are not completely seizure free has important clinical relevance. Additionally, $40 \%$ of patients also showed improved EEG on routine visual analysis during follow-up (Table 3). TSC is characterized by multiple distributed epileptogenic networks, and the improvement in EEG findings probably reflects the result of removing, or at least disrupting, the dominant seizure-generating network, which results in reduced cerebral irritability. There is a remarkable paucity of comparative data on this aspect in the TSC epilepsy surgery literature.

TABLE 4. Univariate comparison of demographic and clinical variables in the ictal video-EEG groups *

\begin{tabular}{lccc}
\hline \multicolumn{1}{c}{ Variable } & Video-EEG Lateralizing & Video-EEG Nonlateralizing & $p$ Value \\
\hline Mean age in yrs & $12.20 \pm 6.89(n=14)$ & $12.19 \pm 5.91(n=20)$ & 0.997 \\
\hline Mean age at seizure onset in mos & $13.14 \pm 21.36(n=14)$ & $6.25 \pm 4.70(n=20)$ & 0.255 \\
\hline Mean presurgery daily seizure frequency & $15.24 \pm 25.78(n=14)$ & $8.49 \pm 12.48(n=20)$ & 0.376 \\
\hline Mean presurgery FSIQ & $73.00 \pm 21.00(n=8)$ & $66.31 \pm 19.83(n=13)$ & 0.481 \\
\hline Mean no. of electrodes & $77.82 \pm 16.93(n=11)$ & $87.36 \pm 16.31(n=14)$ & 0.170 \\
\hline Mean age at the time of surgery in yrs & $5.95 \pm 6.72(n=14)$ & $6.58 \pm 5.74(n=20)$ & 0.780 \\
\hline Mean duration from onset of seizures to surgery in yrs & $4.86 \pm 6.51(n=14)$ & $6.05 \pm 5.62(n=20)$ & 0.582 \\
\hline Mean duration of follow-up in yrs & $5.30 \pm 2.18(n=13)$ & $4.58 \pm 3.01(n=16)$ & 0.461 \\
\hline Mean follow-up FSIQ & $63.00 \pm 1.41(n=2)$ & $62.13 \pm 10.02(n=8)$ & 0.818 \\
\hline No. of patients & & & 0.4867 \\
\hline No. of females & $7 / 14(50.00 \%)$ & $13 / 20(65.00 \%)$ & 0.9999 \\
\hline SPM/ PET concordance with ictal EEG & $7 / 10(70.00 \%)$ & $11 / 16(68.75 \%)$ & 0.6557 \\
\hline SISCOM concordance with ictal EEG & $2 / 7(28.57 \%)$ & $6 / 14(42.86 \%)$ & 0.3544 \\
\hline MEG concordance with ictal EEG & $5 / 6(83.33 \%)$ & $8 / 14(57.14 \%)$ & 0.9999 \\
\hline All functional modalities concordant & $2 / 6(33.33 \%)$ & $6 / 15(40.00 \%)$ & 0.482 \\
\hline ILAE Class 1 outcome & $9 / 14(64.29 \%)$ & $9 / 19(47.37 \%)$ & \\
\hline
\end{tabular}

* Mean values are presented as the mean \pm SD. 


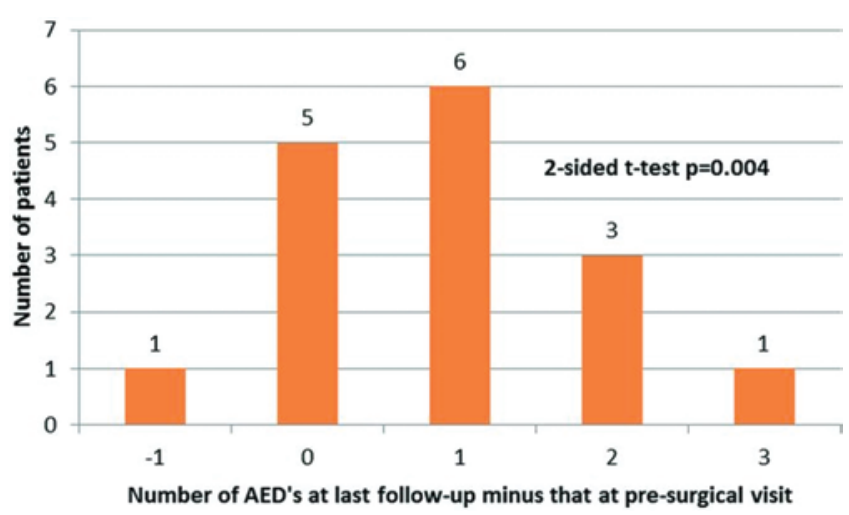

FIG. 3. Change in the AED burden before and after surgery in patients without complete seizure freedom (ILAE Class 2 or worse outcomes). Figure is available in color online only.

Traditionally, surgical candidacy for patients with TSC was limited to those who had a single dominant tuber with concordant neurophysiological data, ${ }^{7,8,15}$ and other children were usually denied resective surgery. This pessimistic outlook was challenged by the group at New York University, who pioneered a multistaged surgical approach based on detection of remaining adjacent epileptogenic tissue or distant secondary epileptogenic foci by another session of invasive monitoring, after a primary resection. ${ }^{3,15}$ In a cohort of 25 children with TSC described by Weiner et al., 22 patients had 3 -stage surgery, including 20 patients with resection of 2 or more tubers and 2 with bilateral resections. ${ }^{14}$ The median durations of invasive monitoring sessions were 7 and 6 days, respectively. At a mean follow-up of 28 months, 17 (68\%) children had Engel Class I outcomes and 6 (24\%) children had Engel Class II outcomes. Importantly, 4 of 5 children who were initially not included as surgical candidates were reported to be seizure free, although follow-up duration was not mentioned..$^{14}$ While this approach is certainly bold and innovative, we used a different approach based on noninvasive and invasive data with conventional postresection electrocorticography to define resection margins. Epidemiologically, these independent case series do not allow comparison of efficacy or safety outcomes. Recently, we have shown prolonged invasive monitoring to be associated with increased incidence of adverse events by $4 \%$ per day after 8 days of monitoring. ${ }^{2}$ Hence, prolonged invasive monitoring on a raw surgical bed may be a factor that has limited wider adaptation of prolonged monitoring with subdural electrodes..$^{14}$ We expect that increasing experience in different institutions with diverse approaches will result in accumulation of outcome and safety data and probably lead to convergence of different surgical approaches to define the optimal strategy.

The meta-analysis by Zhang et al. ${ }^{17}$ identified 3 statistically significant predictors of seizure freedom on univariate analysis: onset of seizures after 1 year of age, lateralization on interictal or ictal EEG, and lobectomy as opposed to tuberectomy. We also found the mean age at onset of seizures to be older in seizure-free patients $(11.19 \pm 17.8$ months) than in the other group $(5.47 \pm 3.62, \mathrm{p}=0.166$; Table 1). Patients with lateralizing video-EEG were found to be more likely to attain seizure freedom (64.29\%) in our study than those with multifocal seizure onset (47.37\%), but this result did not reach statistical significance (OR 2.00 [95\% CI 0.39-10.57], $p=0.482$; Table 4). However, we defined EEG lateralization on the basis of only seizure onset and not interictal epileptiform abnormalities, because children with TSC are highly likely to have bilateral multifocal interictal discharges. ${ }^{4}$ Our study was likely underpowered to detect predictor variables at a statistical significance. Another multicenter retrospective study also reported Engel Class I outcome in $68.6 \%$ of patients with unilateral ictal onset compared with $30.8 \%$ of those with bilateral ictal-EEG abnormalities. ${ }^{10}$ We did not find the type of resective procedure to be a significant predictor of seizure freedom (Fig. 1). Children with TSC require complex and sometimes extensive resections driven by presurgical data, and it is virtually impossible to quantitatively synthesize different studies.

The meta-analysis by Fallah et al. ${ }^{5}$ defined good seizure outcomes as Engel Classes I and II. On univariate analysis, absence of generalized seizure semiology, no or mild developmental delay, unifocal ictal-EEG abnormality, and EEG-MRI concordance were significantly associated with good seizure outcomes. The study lacked a multivariate analysis or study effects adjustment, probably due to small sample sizes of the individual studies, which may represent a publication bias. ${ }^{5}$ The majority of patients in our study had more than one seizure semiology, and for individual patients it was difficult to make decisions about lateralization on a purely clinical basis. We did not find the baseline cognitive status as measured by IQ to be a significant predictor of seizure freedom (Table 1). However, in the 12 patients with available testing, the follow-up IQ was significantly higher in patients with ILAE Class 1 outcome $(66.70 \pm 12.36)$ than in those with ILAE Class 2 or worse outcomes $(56.00 \pm 1.41, p=0.025$, Table 1$)$. This supports the hypothesis that seizure freedom, and probably reduction in antiseizure medications, contributes to improvement in intellectual performance. Furthermore, the improvement in IQ from baseline to that measured during follow-up was found to have a significant positive correlation with patient age (Table 2). While the latter result may be partially due to improved patient performance on psychometric tests with increasing age, we believe these are rather important clinical associations. In this study, followup neuropsychological evaluation was available in only 12 patients with only 2 being in the ILAE Class 2 or worse group. These potential sources of bias prevent a causal inference or clinical utility of this finding at present.

Interestingly, 2 of the predictor variables in the metaanalysis by Fallah et al., ${ }^{5}$ unifocal ictal EEG and EEG-MRI concordance, had significant intrinsic association (Pearson's correlation coefficient $0.37, \mathrm{p}=0.0006$ ). Thus, while the general principle that increased concordance among different modalities predicts better outcome is undisputed, it remains to be objectively demonstrated in patients with TSC. In our study, all patients had bilateral multiple abnormalities on MRI, which represents a more challenging patient population for resective epilepsy surgery. Neither localized seizure onset on scalp EEG nor its concordance with different functional modalities was found to predict 
seizure freedom (Table 1). We believe this is due to small numbers of patients studied with each modality.

There are certain inadvertent limitations in our study due to the limited number of patients and retrospective design. It would have been valuable to assess seizure frequency at different time points in follow-up and model the data using survival methods. However, due to missing data points there is a potential risk for such an analysis being biased toward patients with relatively poorer outcome.

\section{Conclusions}

This study substantiates the evidence that intermediate to long-term seizure freedom can be achieved in TSC patients harboring bilateral multiple brain lesions, with thorough presurgical evaluation and deliberate patient and procedure selection. In this study, $56.8 \%$ of patients achieved complete seizure freedom (ILAE Class 1 outcome) and $86.5 \%$ had ILAE Class 4 or better outcomes after a mean follow-up of 5.68 years. The FSIQ on follow-up was found to be significantly higher in patients with complete seizure freedom $(66.70 \pm 12.36)$ than in those with ILAE Class 2 or worse outcomes $(56.00 \pm 1.41, p=0.025)$. In $62.5 \%$ of patients without complete seizure remission, significant reduction in AED burden was achieved. Forty percent of such patients also showed improvement in EEG findings during follow-up. The creation of a multiinstitutional database would allow for new studies with increased numbers of patients and cumulative results from noninvasive and invasive evaluation to better define the role of each modality and the strategy for optimal surgical decision making.

\section{References}

1. Andrew T, Milinis K, Baker G, Wieshmann U: Self reported adverse effects of mono and polytherapy for epilepsy. Seizure 21:610-613, 2012

2. Arya R, Mangano FT, Horn PS, Holland KD, Rose DF, Glauser TA: Adverse events related to extraoperative invasive EEG monitoring with subdural grid electrodes: a systematic review and meta-analysis. Epilepsia 54:828-839, 2013

3. Bollo RJ, Kalhorn SP, Carlson C, Haegeli V, Devinsky O, Weiner HL: Epilepsy surgery and tuberous sclerosis complex: special considerations. Neurosurg Focus 25(3):E13, 2008

4. Dickerson WW, Hellman CD: Electroencephalographic study of patients with tuberous sclerosis. Neurology 2:248-254, 1952

5. Fallah A, Guyatt GH, Snead OC III, Ebrahim S, Ibrahim GM, Mansouri A, et al: Predictors of seizure outcomes in children with tuberous sclerosis complex and intractable epilepsy undergoing resective epilepsy surgery: an individual participant data meta-analysis. PLoS ONE 8: 53565,2013

6. Franz DN, Bissler JJ, McCormack FX: Tuberous sclerosis complex: neurological, renal and pulmonary manifestations. Neuropediatrics 41:199-208, 2010

7. Guerreiro MM, Andermann F, Andermann E, Palmini A,
Hwang P, Hoffman HJ, et al: Surgical treatment of epilepsy in tuberous sclerosis: strategies and results in 18 patients. Neurology 51:1263-1269, 1998

8. Karenfort M, Kruse B, Freitag H, Pannek H, Tuxhorn I: Epilepsy surgery outcome in children with focal epilepsy due to tuberous sclerosis complex. Neuropediatrics 33:255-261, 2002

9. Kwan P, Arzimanoglou A, Berg AT, Brodie MJ, Hauser WA, Mathern G, et al: Definition of drug resistant epilepsy: consensus proposal by the ad hoc Task Force of the ILAE Commission on Therapeutic Strategies. Epilepsia 51:1069-1077, 2010

10. Madhavan D, Schaffer S, Yankovsky A, Arzimanoglou A, Renaldo F, Zaroff CM, et al: Surgical outcome in tuberous sclerosis complex: a multicenter survey. Epilepsia 48:16251628,2007

11. Major P, Rakowski S, Simon MV, Cheng ML, Eskandar E, Baron J, et al: Are cortical tubers epileptogenic? Evidence from electrocorticography. Epilepsia 50:147-154, 2009

12. Roach ES, DiMario FJ, Kandt RS, Northrup H: Tuberous Sclerosis Consensus Conference: recommendations for diagnostic evaluation. J Child Neurol 14:401-407, 1999

13. Thiele EA: Managing and understanding epilepsy in tuberous sclerosis complex. Epilepsia 51 (Suppl 1):90-91, 2010

14. Weiner HL, Carlson C, Ridgway EB, Zaroff CM, Miles $\mathrm{D}$, LaJoie J, et al: Epilepsy surgery in young children with tuberous sclerosis: results of a novel approach. Pediatrics 117:1494-1502, 2006

15. Weiner HL, Ferraris N, LaJoie J, Miles D, Devinsky O: Epilepsy surgery for children with tuberous sclerosis complex. J Child Neurol 19:687-689, 2004

16. Wieser HG, Blume WT, Fish D, Goldensohn E, Hufnagel A, King D, et al: ILAE Commission Report. Proposal for a new classification of outcome with respect to epileptic seizures following epilepsy surgery. Epilepsia 42:282-286, 2001

17. Zhang K, Hu WH, Zhang C, Meng FG, Chen N, Zhang JG: Predictors of seizure freedom after surgical management of tuberous sclerosis complex: a systematic review and metaanalysis. Epilepsy Res 105:377-383, 2013

\section{Author Contributions}

Conception and design: Mangano. Acquisition of data: Mangano, Arya, Tenney, Greiner, Holland, Leach, Gelfand, Rose, Franz. Analysis and interpretation of data: Arya, Horn, Gelfand, Rozhkov, Fujiwara, Rose. Drafting the article: Arya. Critically revising the article: Mangano, Tenney, Greiner, Holland, Leach, Gelfand, Rose, Franz. Reviewed submitted version of manuscript: Mangano, Arya, Horn, Greiner, Holland, Leach, Gelfand, Rozhkov, Fujiwara, Rose, Franz. Statistical analysis: Horn. Administrative/technical/material support: Franz. Study supervision: Mangano.

\section{Correspondence}

Francesco T. Mangano, Division of Pediatric Neurosurgery, Cincinnati Children's Hospital Medical Center, 3333 Burnet Ave., Cincinnati, OH 45229. email: francesco.mangano@cchmc.org. 